Draft VERSION NOVEMBER 5, 2018

Preprint typeset using $\mathrm{L}^{A} \mathrm{~T} \mathrm{E}$ X style emulateapj v. 11/10/09

\title{
ACCELERATION OF SMALL ASTROPHYSICAL GRAINS DUE TO CHARGE FLUCTUATIONS
}

\author{
A. V. Ivlev ${ }^{1}$, A. Lazarian ${ }^{2}$, V. N. Tsytovich ${ }^{3}$, U. De Angelis ${ }^{4}$, Thiem Hoang ${ }^{2}$, G. E. Morfill $^{1}$ \\ ${ }^{1}$ Max-Planck-Institut für extraterrestrische Physik, 85741 Garching, Germany \\ ${ }^{2}$ Department of Astronomy, University of Wisconsin, Madison, WI 53706, USA \\ ${ }^{3}$ General Physics Institute, Russian Academy of Sciences, 117942 Moscow, Russia \\ ${ }^{4}$ Department of Physical Sciences, University of Naples and INFN, sezione di Napoli, Italy \\ (Dated: November 5, 2018) \\ Draft version November 5, 2018
}

\begin{abstract}
We discuss a novel mechanism of dust acceleration which may dominate for particles smaller than $\sim 0.1 \mu \mathrm{m}$. The acceleration is caused by their direct electrostatic interactions arising from fluctuations of grain charges. The energy source for the acceleration are the irreversible plasma processes occurring on the grain surfaces. We show that this mechanism of charge-fluctuation-induced acceleration likely affects the rate of grain coagulation and shattering of the population of small grains.
\end{abstract}

Subject headings: dust, extinction - ISM: evolution - ISM

\section{INTRODUCTION}

Dust is an important constituent of interstellar medium (ISM), molecular clouds and accretion disks (see Whittet 2003, Draine 2009). It gets involved in many key processes, for instance, it controls heating and cooling of the ISM (see Draine 2003, Tieliens 2005), reveals magnetic fields through grain alignment (see Lazarian 2007 for a review) and interferes with the attempts to measure properties of CMB radiation (see Lazarian \& Finkbeiner 2003, Fraisse et al. 2009).

Small, i.e., less than $\sim 10^{-5} \mathrm{~cm}$ grains, are an important component of the interstellar dust population, with a notable fraction of very small grains - Polycyclic Aromatic Hydrocarborn (PAH) particles, which are essentially large molecules (see Leger \& Puget 1984). In what follows, due to the reasons that are explained in $\S 5$, we do not directly address $\mathrm{PAH}$ particles, but our approach to the small grain acceleration may be extended to this important population of grains.

Most properties of grains, including light extinction, electron photoemission, and chemical activity depend not only on grain chemical composition, but also on their sizes. In astrophysical media, these sizes are affected by grain-grain collisions. The minimal velocities of grains are determined by their Brownian motion corresponding to the temperature of the ambient gas. Large-scale hydrodynamic motions associated with turbulence can make grains move faster (see Draine 1985).

Since most astrophysical media are magnetized and grains are charged the hydrodynamic treatment of acceleration is frequently not adequate. A proper treatment of the grain acceleration through the interaction of charged grains with magnetohydrodynamic (MHD) turbulence has been developed recently (Lazarian \& Yan 2002, Yan \& Lazarian 2003, Yan, Lazarian \& Draine

\footnotetext{
E-mail: ivlev@mpe.mpg.de

1 The information about these grains is being obtained through the transient heating of grains by UV photons. Indeed, after absorbing a photon a grain gets heated to rather high temperature, inducing emission of infrared photons (see Allamandola et al. 1989). The PAHs were invoked by Draine \& Lazarian (1998) to explain the anomalous emission of observed in the range of $10-100 \mathrm{GHz}$ (see also Hoang, Draine \& Lazarian 2010).
}

2004, Yan 2009). This treatment makes extensive use of the advances of compressible MHD turbulence (see Cho \& Lazarian 2002, 2003) and provides the mathematical formalism of the second-order Fermi acceleration of charged grains interacting with MHD turbulence.

However, the acceleration mechanisms based on the MHD interaction of turbulence and charged grains exhibit acceleration rates that decrease as dust particles get smaller. This decrease arises from the fact that the Larmor radius of charged grains becomes smaller with the decrease of grain mass and, correspondingly, grains have to interact with smaller, i.e., less powerful, turbulent fluctuations. In addition, compressible fluctuations, i.e., fast modes, which were identified in Yan \& Lazarian (2003) with the most efficient acceleration, get suppressed at the small scales due to plasma damping, while the Alfvenic mode gets inefficient for acceleration at small scale due to anisotropy (see Yan \& Lazarian 2003 for more discussion). The acceleration of grains with sizes less than $\sim 10^{-5} \mathrm{~cm}$ becomes rather inefficient for most media discussed in Yan \& Lazarian (2003), Yan, Lazarian \& Draine (2004, henceforth YLD04).

Are there other mechanisms of dust acceleration which dominate for grains smaller than $10^{-5} \mathrm{~cm}$ ? One can expect that dust-plasma interactions may be important for such grains. This paper presents a novel promising acceleration mechanism based on grain-grain Coulomb collisions in the presence of grain charge fluctuations. This mechanism utilizes intrinsic non-equilibrium nature of the dusty astrophysical plasmas. In addition to acting on the small grains, the mechanism may drive the acceleration of larger grains whenever the MHD acceleration mechanism is suppressed (e.g., when MHD turbulence is damped).

In what follows, we introduce major timescales characterizing dynamics of charged interstellar grains in $\S 2$, present the mechanism of small grain acceleration associated with charge fluctuations during their Coulomb collisions in $\S 3$, calculate the acceleration for grains of a given size distribution in $\S 4$, analyze implications of the effect for interstellar phases in $\S 5$, discuss the importance of our results in $\S 6$, and summarize them in $\S 7$. 


\section{DUST INTERACTIONS IN INTERSTELLAR GAS}

\subsection{Dust damping time}

Interactions of dust with the ambient gas present the primary mechanism of dissipating streaming motions of grains. The damping rate of translational motion arising from the interaction with neutral gas is essentially the inverse time for collisions with the mass of the gas equal that of a grain (Purcell 1969),

$$
\tau_{d n}^{-1}=2 \sqrt{\frac{2}{\pi}} \frac{n_{n}}{a \rho_{d}}\left(m_{n} k_{\mathrm{B}} T_{n}\right)^{1 / 2},
$$

where $m_{n}, n_{n}$, and $T_{n}$ are the mass, volume density, and temperature of neutrals, $\rho_{d}$ is the mass density of dust grains and $a$ is their radius.

When the ionization is sufficiently high, the interaction of charged grains with a plasma becomes important (Draine \& Salpeter 1979). The ion-grain cross section due to long-range Coulomb force is larger than the atomgrain cross section. As a result, the rate of translational motion damping gets modified (Draine \& Salpeter 1979). For subsonic motions the effective damping rate is renormalized, $\tau_{\text {damp }}^{-1}=\alpha \tau_{d n}^{-1}$, with the following renormalizing factor:

$$
\begin{array}{r}
\alpha=1+\frac{n_{\mathrm{H}}}{2 n_{n}} \sum_{i} x_{i}\left(\frac{e^{2}}{a k_{\mathrm{B}} T_{i}}\right)^{2}\left(\frac{m_{i}}{m_{n}}\right)^{1 / 2} \sum_{Z} Z^{2} f(Z) \\
\times \ln \frac{3}{2 \sqrt{\pi}} \frac{\left(k_{\mathrm{B}} T_{i}\right)^{3 / 2}}{|Z| e^{3}\left(x n_{\mathrm{H}}\right)^{1 / 2}} .
\end{array}
$$

Here $x_{i}$ is the abundance of ion $i$ (relative to hydrogen) with mass $m_{i}$ and temperature $T_{i}, x=\sum_{i} x_{i}$, and $f(Z)$ is the grain charge distribution function (see Hoang, Draine \& Lazarian 2010). When the grain velocity $v_{d}$ relative to the gas becomes supersonic the dust interactions with the plasma is diminished, and the damping rate in this case is renormalized due to the gas-dynamic correction (Purcell 1969),

$$
\alpha=\left(1+\frac{9 \pi}{128} \frac{v_{d}^{2}}{C_{\mathrm{s}}^{2}}\right)^{1 / 2},
$$

where $C_{\mathrm{s}}=\sqrt{k_{\mathrm{B}} T / m_{n}}$ is the sound speed.

The Larmor rate for a grain is $\tau_{\mathrm{L}}^{-1}=\left|Z_{0}\right| e B / m_{d} c$, where $Z_{0}(a)$ is the mean grain charge and $B$ is the magnetic field strength. If $\tau_{\mathrm{L}}$ is larger than $\tau_{\text {damp }}$ the effect of magnetic field on dust dynamics is negligible.

\subsection{Grain Coulomb interactions}

Grains can interact with each other through Coulomb forces if the Debye screening length is larger than the distance between grains. In Figure 11 we show the ratio of the Debye radius $\lambda_{\mathrm{D}}$ to the separation $d_{a}$ between the grain of size $a$ and grains of a larger size, calculated for three different idealized interstellar phases: the cold neutral medium (CNM), warm neutral medium (WNM), warm ionized medium (WIM). Table 1 presents physical parameters for such environments [including also reflection nebula $(\mathrm{RN})$ and photodissociation region $(\mathrm{PDR})]$, where $n_{\mathrm{H}}$ is the hydrogen density, $T$ and $T_{d \mathrm{~s}}$ are the gas temperature and dust material temperature, respectively, $n\left(\mathrm{H}^{+}\right), n\left(\mathrm{M}^{+}\right)$and $n\left(\mathrm{H}_{2}\right)$ are ion hydrogen density, ion metal density and molecular hydrogen density,

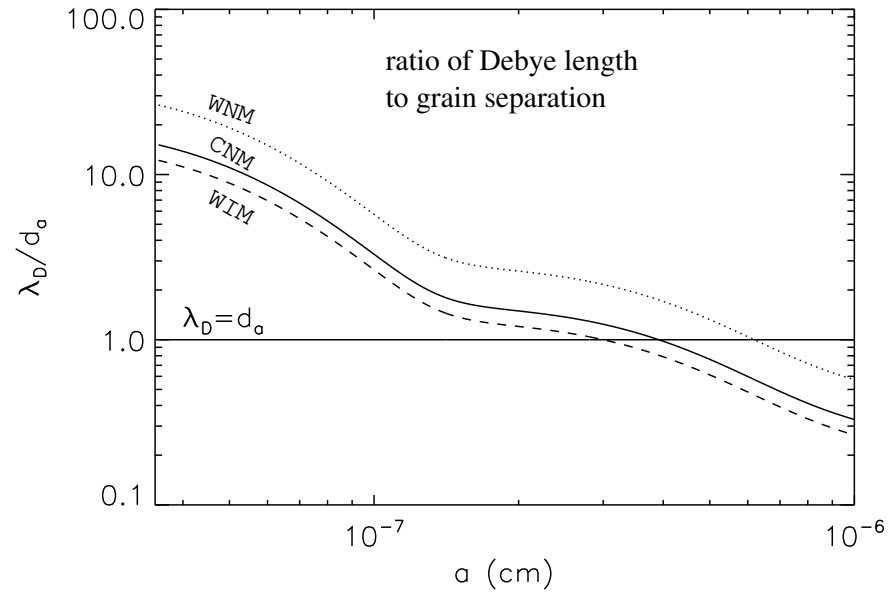

FIG. 1. - The ratio of the Debye screening length, $\lambda_{\mathrm{D}}$, to the distance $d_{a}$ between grains larger than a given size $a$.

TABLE 1

IDEALIZED EnVIRONMENTS FOR THE Interstellar Matter

\begin{tabular}{llllll}
\hline \hline \multicolumn{1}{c}{ Parameter } & CNM & WNM & WIM & RN & PDR \\
\hline$n_{\mathrm{H}}\left(\mathrm{cm}^{-3}\right)$ & 30 & 0.4 & 0.1 & $10^{3}$ & $10^{5}$ \\
$T(\mathrm{~K})$ & 100 & 6000 & 8000 & 100 & 1000 \\
$T_{d \mathrm{~m}}(\mathrm{~K})$ & 20 & 20 & 20 & 40 & 80 \\
$x_{\mathrm{H}}=n\left(\mathrm{H}^{+}\right) / n_{\mathrm{H}}$ & 0.0012 & 0.1 & 0.99 & 0.001 & 0.0001 \\
$x_{\mathrm{M}}=n\left(\mathrm{M}^{+}\right) / n_{\mathrm{H}}$ & 0.0003 & 0.0003 & 0.001 & 0.0002 & 0.0002 \\
$y=2 n\left(\mathrm{H}_{2}\right) / n_{\mathrm{H}}$ & 0. & 0. & 0. & 0.01 & 0.01 \\
& & & & & \\
\hline \hline
\end{tabular}

respectively. To represent grain charge we use the charging data from Hoang et al. (2010).

It is clear that for grains smaller than $\sim 3 \times 10^{-7} \mathrm{~cm}$ the grains do interact electrostatically with their larger neighbors. As a result of charge fluctuations, the dust particles experience forces which can accelerate them.

We choose to depict distances to the grains of the size larger than the given grain size, because such interactions will be most important in terms of momentum transfer. Naturally, larger grains will be still interacting with smaller ones and therefore there should be no sharp cutoff for the grains to stop being accelerated by the chargefluctuation mechanism.

\section{MECHANISM OF DUST ACCELERATION}

\subsection{Physics of the process}

Astrophysical grains are known to be charged (see Draine \& Sutin 1987). The grain charges are not constant - they fluctuate around an equilibrium value $Q_{0}=$ $e Z_{0}$. In a plasma without external radiation $Q_{0}$ is negative, because electrons are much faster than ions (in the presence of sufficiently intense external UV radiation it becomes positive, see Goree 1994). In general, one needs to deal with a distribution of charges (see Weingartner \& Draine 2001, Hoang et al. 2010).

It is possible to demonstrate that the mutual Coulomb collisions between dust particles with fluctuating charges do not conserve the kinetic energy of the particles. As 
the energy is being taken from the fluctuations the kinetic energy of the particles increase in a stochastic way (see de Angelis et al. 2005, Ivlev et al. 2005, henceforth I05) - a process similar to second-order Fermi acceleration, where the role of randomly moving magnetic mirrors is played by the randomly fluctuating electric forces exerted by other particles.

The physical mechanism behind such stochastic heating is easy to understand. Each particle creates in its vicinity a fluctuating electric field, so that the superposition of these fluctuations creates a stochastic background which can be stronger than the fluctuations induced by the plasma itself. An individual dust particle in such a random environment experiences fluctuating electric force and hence performs a random walk in the momentum space. This naturally results in the stochastic acceleration of dust particles.

Below we elaborate on this mechanism. We first consider binary interactions (collisions) between particles with fluctuating charges, and demonstrate that such interactions result in the effective particle acceleration. Next, we employ the kinetic approach and show that in large particle ensembles the stochastic acceleration in binary collisions can result in the exponential growth of the particle kinetic energy (temperature). We stress that we consider collisions between grains only for illustrative purposes, as it is one way to show that energy of grains do change as a result of charge fluctuations. At the same time, the essential element of the acceleration process is the fluctuation of electric force acting on a particle, which occurs even if grains are not directly colliding.

\subsection{Stochastic acceleration in binary collisions}

We consider dilute dust clouds and hence only focus on the binary interactions between grains. Binary collisions can be conveniently studied in terms of the center-ofmass and relative coordinates. Below we consider grains of the same mass, $m_{d 1}=m_{d 2}\left(=m_{d}\right)$, although all results can be straightforward generalized for arbitrary mass ratio (Ivlev et al. 2004): e.g., for $m_{d 1} / m_{d 2} \ll 1$ one should substitute the mass of light particle instead of the reduced mass $\left(\frac{1}{2} m_{d}\right)$. For a pair of particles with momenta $\mathbf{p}_{1}$ and $\mathbf{p}_{2}$, the center-of-mass and relative momenta are $\mathbf{p}_{\mathrm{c}}=\frac{1}{2}\left(\mathbf{p}_{1}+\mathbf{p}_{2}\right)$ and $\mathbf{p}_{\mathrm{r}}=\mathbf{p}_{1}-\mathbf{p}_{2}$, respectively. The kinetic energy of the pair can be expressed via $p_{\mathrm{c}}$ and $p_{\mathrm{r}}$ as follows: $p_{1}^{2} / 2 m_{d}+p_{2}^{2} / 2 m_{d}=p_{\mathrm{c}}^{2} / m_{d}+p_{\mathrm{r}}^{2} / 4 m_{d} \equiv \varepsilon_{\mathrm{c}}+\varepsilon_{\mathrm{r}}$. The center-of-mass momentum and hence the energy $\varepsilon_{\mathrm{c}}$ are conserved during the collision (here we neglect momentum exchange due to collisions with electrons and ions), whereas the relative momentum is changed,

$$
\mathbf{p}_{\mathrm{c}}^{\prime}=\mathbf{p}_{\mathrm{c}} ; \quad \mathbf{p}_{\mathrm{r}}^{\prime}=\mathbf{p}_{\mathrm{r}}+\mathbf{q} .
$$

For constant charges $\varepsilon_{\mathrm{r}}$ is obviously conserved during the collision, but when charges vary $\varepsilon_{\mathrm{r}}$ varies as well. Thus, in the presence of charge fluctuations the exchange of the relative momentum can be divided into the elastic and inelastic parts, $\mathbf{q}=\mathbf{q}_{0}+\delta \mathbf{q}$. The elastic part keeps the magnitude of the relative momentum constant, $\left|\mathbf{p}_{\mathrm{r}}+\mathbf{q}_{0}\right|=\left|\mathbf{p}_{\mathrm{r}}\right|$, so that the energy variation after the collision is $\delta \varepsilon_{\mathrm{r}}=\left(\mathbf{p}_{\mathrm{r}}+\mathbf{q}_{0}\right) \cdot \delta \mathbf{q} / 2 m_{d}+(\delta \mathbf{q})^{2} / 4 m_{d}$. The inelastic momentum exchange $\delta \mathbf{q}$ is generally a function of $\mathbf{p}_{\mathrm{c}}$ and $\mathbf{p}_{\mathrm{r}}$ and is determined by the stochastic properties of charge fluctuations.
In order to calculate the energy variation, we suppose that for different particles the charge fluctuations are uncorrelated, so that for a pair of particles with charges $Q_{1}(t)=Q_{0}+\delta Q_{1}(t)$ and $Q_{2}(t)=Q_{0}+\delta Q_{2}(t)$ we have $\left\langle\delta Q_{1}(t+\tau) \delta Q_{2}(t)\right\rangle=0$. Assuming that $\left|\delta Q / Q_{0}\right| \ll 1$, the inelastic momentum exchange due to the collision can be calculated as $\delta \mathbf{q} \approx \int\left[\delta Q_{1}(t)+\delta Q_{2}(t)\right] \nabla \varphi_{0} d t$, where $\varphi_{0}(r)$ is the potential of the equilibrium charge $Q_{0}$ and the integration is performed along the (equilibrium) collision trajectory $\mathbf{r}(t)$. The resulting energy variation is obtained by averaging over the fluctuations, i.e.,

$$
\delta \varepsilon_{\mathrm{r}} \equiv \frac{\left\langle(\delta \mathbf{q})^{2}\right\rangle}{4 m_{d}}=\frac{1}{2 m_{d}}\left\langle\int \delta Q \nabla \varphi_{0} d t \int \delta Q^{\prime} \nabla \varphi_{0}^{\prime} d t^{\prime}\right\rangle .
$$

To calculate $\delta \varepsilon_{\mathrm{r}}$ we also assume $\left|\delta Q / Q_{0}\right| \ll 1$ and therefore can use the following charge auto-correlation function (Matsoukas \& Russell 1997):

$$
\langle\delta Q(t+\tau) \delta Q(t)\rangle=\sigma_{Q}^{2} \mathrm{e}^{-\nu_{\mathrm{ch}}|\tau|} .
$$

Here $\sigma_{Q}^{2}$ is the charge dispersion and $\nu_{\mathrm{ch}}$ is the "charging frequency" (which plays the role of the scale parameter in the charge fluctuations spectrum). The assumption about small charge fluctuations simplifies significantly the derivation of Eq. (4), and also allows us to treat the charge as a continuous variable and hence employ Eq. (5).

There are several mechanisms that can result in the fluctuations (Yan et al. 2004, Khrapak et al. 1999). In this paper, for the sake of convenience we focus on the most elementary charging process which is due to discreteness of the elementary charge. Usually, this is a Gaussian process with $\sigma_{Q}^{2}=\frac{1+z}{z(2+z)}\left|e Q_{0}\right|$ and $\nu_{\mathrm{ch}}=\frac{1}{\sqrt{2 \pi}}(1+z) a \omega_{\mathrm{p} i}^{2} / v_{T_{i}}$ (Matsoukas \& Russell 1997), where $\omega_{\mathrm{p} i}=\sqrt{4 \pi e^{2} n_{i} / m_{i}}$ is the ion plasma frequency, $v_{T_{i}}=\sqrt{k_{\mathrm{B}} T_{i} / m_{i}}$ is the ion thermal velocity, and $z=$ $e\left|Q_{0}\right| / a k_{\mathrm{B}} T_{i}$ is the dimensionless charge of dust particle of the radius $a$ (for different gases, $z=1-3$ ) (Tsytovich 1997, Fortov et al. 2005). Note that other charging processes can also play an important role (in particular, the photoelectric emission) and require separate careful consideration.

One can identify two limiting collisional regimes associated with the charge fluctuations. In the limit of rapid fluctuations the charges vary many times in each act of binary collisions between dust particles. This regime corresponds to $\nu_{\mathrm{ch}} \tau_{\text {int }} \gg 1$, where $\tau_{\text {int }}\left(v_{d}\right)$ is the characteristic timescale of the dust-dust interaction during the collision. By employing Eq. (5), we readily obtain from Eq. (4) the following limiting expression for the energy variation:

$$
\delta \varepsilon_{\mathrm{r}} \approx \frac{\sigma_{Q}^{2}}{m_{d} \nu_{\mathrm{ch}}} \int\left|\frac{d \varphi_{0}}{d r}\right|^{2} d t+O\left(\nu_{\mathrm{ch}}^{-2}\right) .
$$

Equation (6) shows that in this limit each collision between dust particles results in the increase of their kinetic energy (I05).

The interaction timescale $\tau_{\text {int }}$ is determined by the range of the fluctuating field. In contrast to the average field, which is screened at the plasma Debye length $\lambda_{\mathrm{D}}$, the screening of the fluctuating field occurs at the 
so-called "plasma flux" scale $\lambda_{\text {flux }}$ (see, e.g., Tsytovich et al 2008). The physical meaning of $\lambda_{\text {flux }}$ is rather simple: While the screening of the average field is characterized by the timescale $\sim \omega_{\mathrm{p} i}^{-1}$ and occurs at the length scale $\sim v_{T_{i}} / \omega_{\mathrm{p} i}=\lambda_{\mathrm{D}}$, the relevant timescale for charge fluctuations is $\sim \nu_{\mathrm{ch}}^{-1}$ and hence they are screened at the length scale $\sim \lambda_{\text {flux }}=v_{T_{i}} / \nu_{\text {ch }}$. Thus, the dust interaction timescale is $\tau_{\text {int }} \sim \lambda_{\text {flux }} / v_{d}$ and therefore the condition of rapid fluctuations is reduced to $v_{T_{i}} / v_{d} \gg 1$, i.e., it is generally satisfied for the subsonic dust.

In the opposite limit of slow fluctuations the characteristic time of the charge variations is much longer than the collision time. This regime is completely analogous to "regular" Fermi acceleration - with equal probability, each collision results in energy gain or loss, but the collisions resulting in the energy gain are more frequent. In this case, after averaging over many collisions, we also obtain net energy growth. Analysis of this regime will be presented elsewhere.

\subsection{Evolution of dust kinetic temperature}

In order to understand the effect of the energy variation in binary dust-dust collisions [given by Eq. (6)] on the mean kinetic energy of the whole dust ensemble one should employ the kinetic approach. The kinetics is described in terms of the velocity distribution function $f_{d}(\mathbf{p}, t)$. There are two principal contributions to the dust kinetics - one is due to the mutual dust collisions and another because of dust interactions with the ambient gas. For simplicity, we first consider the idealized situation for the second contribution and only take into account the interactions with neutrals of temperature $T_{n}$ (we generalize this idealized setup for more realistic environments in $\S 5$ ).

The resulting kinetic equation has the following form:

$$
\frac{d f_{d}}{d t}=\mathrm{St}_{d d} f_{d}+\mathrm{St}_{d n} f_{d}
$$

where $\mathrm{St}_{d d}$ and $\mathrm{St}_{d n}$ denote the collision operators (integrals) describing the dust-dust and dust-gas interactions, respectively. Although the analysis of Eq. (7) can be performed for arbitrary $f_{d}(\mathbf{p})$, for the sake of convenience we assume that dust particles have a Maxwellian velocity distribution $f_{M}(\mathbf{p})$. Then the equation for evolution of mean kinetic energy (kinetic temperature) $k_{\mathrm{B}} T_{d}(t)=$ $\frac{1}{3} \int\left(p^{2} / m_{d}\right) f_{\mathrm{M}} d \mathbf{p}$ is obtained by taking the second moment of Eq. (7),

$$
\dot{T}_{d}=\frac{1}{3} \int \frac{p^{2}}{m_{d}}\left(\mathrm{St}_{d d} f_{\mathrm{M}}+\mathrm{St}_{d n} f_{\mathrm{M}}\right) d \mathbf{p} .
$$

The integrals in Eq. (8) are calculated separately in Appendices $\mathrm{A}$ and B. Equation (A1) yields the second integral (contribution of dust-neutral interactions), whereas the first integral describing the dist-dust collisions is given by Eq. (B4). The latter is determined by the kinetic coefficient $\mathcal{A}_{d d}$, which is calculated in Appendix C. Eq. (C1). By substituting this in Eq. B4 (and taking into account that the coefficient $\mathcal{B}_{d d}$ can be neglected, see Appendix B], we derive the following equation for the kinetic temperature of dust (I05):

$$
\dot{T}_{d}=\frac{\sigma_{Q}^{2} \omega_{\mathrm{p} d}^{2}}{Q_{0}^{2} \nu_{\mathrm{ch}}} T_{d}-2 \tau_{d n}^{-1}\left(T_{d}-T_{n}\right),
$$

where $\omega_{\mathrm{p} d}=\sqrt{4 \pi Q_{0}^{2} n_{d} / m_{d}}$ is the dust plasma frequency.

Without charge fluctuations, when the collisions between dust particles conserve the energy, the equilibrium temperature of dust is determined by interactions with the ambient gas, so that $T_{d}=T_{n}$. Random charge fluctuations provide an additional energy source, and if the coefficient of the first (source) term in the r.h.s. of Eq. (9) exceeds the damping rate $2 \tau_{d n}^{-1}$, then the dust temperature grows exponentially with time.

The kinetic coefficient $\mathcal{A}_{d d}$ (Appendix $\mathrm{C}$ ) is linearly proportional to the maximum scattering angle, which we set equal to unity. Hence, we implicitly supposed that there are sufficiently small impact parameters $\rho$ that ensure scattering at large angles, $\chi \gtrsim 1$. However, since the lower bound of impact parameters is limited by the particle radius, $\rho \gtrsim a$, this assumption is only valid if the kinetic temperature is below a certain critical value $T_{d}^{c r}$. From the relation $\chi \sim Q_{0}^{2} / \rho T_{d}$ (Lifshitz \& Pitaevskii 1981) we readily deduce,

$$
T_{d}^{\mathrm{cr}} \sim \frac{Q_{0}^{2}}{a} \equiv \frac{\left|Q_{0}\right|}{e} z T_{i},
$$

so that the actual value of the maximum scattering angle is equal to $T_{d}^{\mathrm{cr}} / T_{d}$. Therefore, the exponential temperature growth described by Eq. (9) proceeds until $T_{d}$ reaches the critical value $T_{d}^{\mathrm{cr}}\left(\gg T_{i}\right)$. At larger temperatures the source term in Eq. (9) tends to a constant value (which is obtained by replacing $T_{d}$ with $T_{d}^{\mathrm{cr}}$ ). The temperature growth crosses over to linear and eventually gets saturated due to the friction, which determines the ultimate temperature of dust.

\section{SIZE-DEPENDENCE OF KINETIC TEMPERATURE FOR ASTROPHYSICAL GRAINS}

Let us now determine the conditions (i) when the exponential heating described by Eq. (9) can set in, (ii) what is the magnitude of the ultimate kinetic temperature that can be reached by astrophysical grains due to the stochastic heating, and (iii) what are the characteristic timescales to reach this temperature. We shall assume an isothermal $\left(T_{n} \approx T_{e} \approx T_{i}\right)$ quasineutral $\left(n_{e} \approx n_{i}\right)$ plasma with arbitrary value of the ionization fraction $n_{i} / n_{n}$. Furthermore, we naturally consider dust as the "test species" which does not change the global energy balance, and therefore do not take into account the feedback the dust has on the ISM plasma.

(i) By substituting the above defined charge dispersion $\sigma_{Q}$ as well as frequencies $\omega_{\mathrm{p} d}, \nu_{\mathrm{ch}}$, and $\tau_{d n}^{-1}$ in Eq. (9) we obtain the heating condition,

$$
\frac{\left|Q_{0}\right| n_{d}}{e n_{i}}>\frac{16}{3} z(2+z) n_{n} a^{3} .
$$

Then, by using the definition of $z$ and taking into account that in an isothermal hydrogen plasma $z \approx 2.5$ (Tsytovich 1997, Fortov et al. 2005), we reduce the condition for the dust heating to the following simple formula:

$$
a^{2} \lesssim 0.1 \lambda_{\mathrm{D}}^{2} \frac{n_{d}(a)}{n_{n}} .
$$

This inequality shows that the heating occurs for particles below a certain critical size. The mechanism of the stochastic acceleration is effective for collisions with particles of size $\gtrsim a$, so that the relevant dust density to 
be substituted in Eq. (10) is determined by the integral $n_{d}(a)=\int_{a}^{\infty}\left(d n_{d} / d a\right) d a$, where $d n_{d} / d a$ is the appropriate size distribution. Usually $d n_{d} / d a$ decreases rather steeply and therefore the critical size should have a weak dependence on the parameters of the ISM.

For the estimate, let us assume the MRN size distribution for dust (Mathis, Rumpl, \& Nordsieck 1977) valid for the size range between a few dozens of $\AA$ and a few tenths of $\mu \mathrm{m}$ :

$$
\frac{d n_{d}}{d a}=-n_{\mathrm{H}} A_{\mathrm{MRN}} a^{-3.5},
$$

where $A_{\mathrm{MRN}} \sim 10^{-25} \mathrm{~cm}^{2.5}$ (Draine \& Lee 1984). This yields $n_{d}(a) / n_{\mathrm{H}} \sim 0.3 A_{\mathrm{MRN}} a^{-2.5}$. Taking $m_{i} \sim 10^{-24} \mathrm{~g}$, $n_{i} \sim 1 \mathrm{~cm}^{-3}, T_{i} \sim 0.1 \mathrm{eV}$, and assuming also $n_{n} \approx$ $n_{\mathrm{H}}$, we get the critical diameter of $\sim 0.3 \mu \mathrm{m}$, i.e., the heating can already be triggered in the submicron range. It is noteworthy that the critical size also has a weak dependence on the stochastic properties of the charge fluctuations. For instance, from Eq. (9) one can readily deduce that the scaling of the critical size on the charging frequency is $\propto \nu_{\mathrm{ch}}^{-0.2}$.

(ii) In order to determine the ultimate temperature $T_{d}^{\infty}$ that can be reached, we recall that when $T_{d}$ exceeds the critical value $T_{d}^{\mathrm{cr}}$, the source term in Eq. (9) is saturated at a constant value. Hence, we have $T_{d}^{\infty} \sim 0.1\left(\lambda_{\mathrm{D}} / a\right)^{2}\left(n_{d} / n_{n}\right) T_{d}^{\mathrm{cr}}$. By using the definition of $z$, the critical temperature can be rewritten as $T_{d}^{\mathrm{cr}}=z^{2}\left(T_{i} / e\right)^{2} a$. Then we finally get the following estimate for the ultimate kinetic temperature of grains:

$$
\frac{T_{d}^{\infty}}{T_{i}} \sim \frac{n_{i}}{n_{n}} \frac{\lambda_{\mathrm{D}}^{4} n_{d}(a)}{a} .
$$

Thus, the increase of the dust temperature relative to the temperature of gas depends very strongly on the grain size. Assuming again the MRN distribution for $n_{d}(a)$ we get $T_{d}^{\infty} / T_{i} \propto a^{-3.5}$. The corresponding RMS velocity of grains, $v_{T_{d}}^{\infty}=\sqrt{k_{\mathrm{B}} T_{d}^{\infty} / m_{d}}$, scales as $\propto a^{-3.25}$, whereas the dependence on the plasma parameters is weaker, $\propto$ $T_{i}^{3 / 2} n_{i}^{-1 / 2}$. For the parameters used above, a $30 \mathrm{~nm}$ dust particle can reach the kinetic temperature which is $5-6$ orders of magnitude higher than $T_{i}$.

(iii) The heating timescale $\tau_{\infty}$ (i.e., when $T_{d}^{\infty}$ is reached) is determined by the stage of linear temperature growth. This can be straightforwardly obtained from Eq. (9),

$$
\tau_{\infty} \sim\left(\frac{e}{Q_{0}}\right)^{2} \frac{m_{d}}{m_{i}} \frac{n_{i}^{2} \lambda_{\mathrm{D}}^{4}}{n_{n} v_{T_{i}}} .
$$

The heating timescale is independent of the plasma density and has rather weak dependence on other parameters, $\tau_{\infty} \propto a n_{n}^{-1} T_{i}^{-1 / 2}$. This estimate yields heating timescale of several Myr for $30 \mathrm{~nm}$ grains.

The formalism above is rather general and is applicable to any astrophysical media, including accretion disks, circumstellar regions and the ISM. In the next section we focus our attention on the ISM dust acceleration.

\section{IMPLICATIONS FOR ISM DUST}

The ISM dust acceleration is extremely important process for shattering and coagulation of dust (see Hirashita

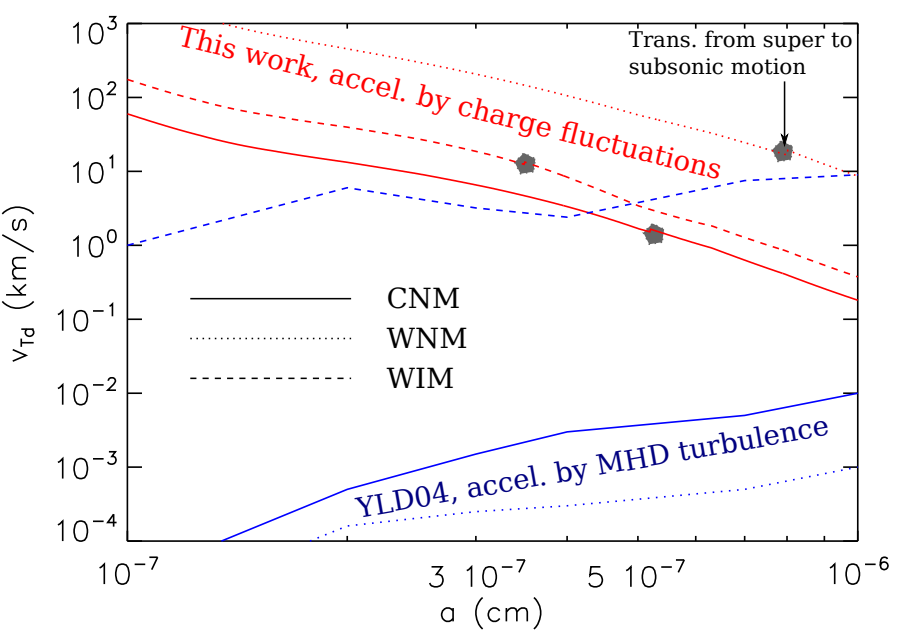

FIG. 2.- RMS velocity of dust grain for the parameters of ISM phases given in Table 1. The grain size distribution function from Weingartner \& Draine (2001) is adopted. Diamond symbols denote the transition from supersonic to subsonic motion of dust.

\& Yan 2009). We discuss both subsonic and supersonic motion of dust. In Eq. (D4) (Appendix D) we rewrite Eq. (12) in terms of the RMS velocity of accelerated grains assuming the MRN dust size distribution, Eq. (11)

$$
v_{T_{d}}^{\infty} \approx \frac{6 \times 10^{2}}{\left(\alpha n_{i}\right)^{1 / 2}}\left(\frac{T_{i}}{100 \mathrm{~K}}\right)^{3 / 2}\left(\frac{a}{10^{-6} \mathrm{~cm}}\right)^{-3.25} \mathrm{~cm} / \mathrm{s},
$$

where where $n_{i}$ is in $\mathrm{cm}^{-3}$ and the renormalizing factor $\alpha$ for the damping rate in the subsonic and supersonic regimes is given by Eq. (2) and (3), respectively. Note that in the latter case $\alpha$ is the function of dust velocity and hence Eq. (13) should be resolved for $v_{T_{d}}^{\infty}$.

In Figure 2 we compare the mechanism presented in the paper with the calculations of the velocities of interstellar grains obtained in YLD04. We see that for sufficiently large grains the acceleration due to MHD fluctuations is stronger. At the same time, the new mechanism of acceleration associated with charge fluctuations is more efficient for $a \lesssim 10^{-5} \mathrm{~cm}$.

The efficiency of the charge-fluctuation-induced acceleration increases for smaller grains. However, the limitation of our treatment above is that the grain charging was considered as a continuous process [see Eq. (5)]. Formally, this approach is not applicable to the situation when the mean grain charge $Q_{0}$ is so small that each act of losing or acquiring an elementary charge results in substantial discrete fluctuations. However, we believe that even in this case we may get right order of magnitude estimate of grain acceleration. Further research with Monte-Carlo simulations instead of using FokkerPlank approach should test the latter conjecture.

Comparing the Figures 1 and 2 we see the limited nature of our quantitative calculations. For grains larger than $\sim 3 \times 10^{-7} \mathrm{~cm}$ the interactions decrease due to the less efficient momentum deposition as grains mostly feel the electric field of grains smaller than their size. On the other hand, the charge fluctuations become large for grains appreciably smaller than this size and this makes the treatment of the problem with the Fokker-Planck equation not precise. Nevertheless, even taking into ac- 
count the pilot nature of our estimates it is clear that the process of acceleration is potentially very important for small grains.

\section{DISCUSSION}

We showed that there exists a novel powerful mechanism of charge-fluctuation-induced acceleration operating in astrophysical plasmas. This mechanism, which occurs to be more efficient for smaller grains, is based on the charge fluctuations that grains experience during the mutual Coulomb collisions. As the result, the kinetic energy of grains is not conserved in the collisions, which causes the stochastic heating analogous to the secondorder Fermi acceleration.

This acceleration mechanism is a generic plasma process which can operate in very different environments ranging from the ISM to laboratory gas discharges. It is a remarkable manifestation of the intrinsic non-equilibrium (energetic openness) of dusty plasmas (Tsytovich 1997, Fortov 2005): Even if electrons and ions themselves were in detailed equilibrium, their absorption and the subsequent recombination on dust grains cannot be balanced by the corresponding inverse process (of the ionization and emission from grains) due to its apparent inefficiency - temperatures necessary for that should be enormously high. Therefore, the process of dust charging goes only in one direction, resulting in the imbalanced plasma flux on the grain surface. The dust dynamics gets coupled to the charging process due to charge fluctuations (which are inevitable due to the discreteness of the elementary charge). Thus, in terms of the energy balance, the mechanism of charge-fluctuation-induced acceleration is based on the conversion of the energy flux (associated with the plasma flux on grains) into the kinetic energy of dust.

One should point out that for typical ISM conditions the situation is very different from the equilibrium one: The plasma temperature is much larger that the dust material temperature $\left(T_{d \mathrm{~m}}\right)$ due to efficient radiative cooling of the dust. Moreover, the ions and electrons are usually produced by the ISM UV and/or cosmic ray ionization. Grains themselves emit photoelectrons interacting with UV photons. All these non-equilibrium effects can alter charge fluctuations and therefore additionally contribute to our acceleration mechanism.

In particular environments, e.g., within quiescent dark clouds and protoplanetary disks with suppressed turbulence, the mechanism we discussed in the paper may be dominant for grains substantially larger than $10^{-5} \mathrm{~cm}$. However, we notice the rapid decrease of the mechanism efficiency with the grain size.

The treatment presented in this paper is limited in two respects. First, the Fokker-Planck approach presumes relatively small energy variation (6) occurring in each Coulomb collision due to charge fluctuations. If this variation becomes large, such approach (describing the evolution of the velocity distribution function) is no longer applicable. On the other hand, the mean energy evolution - which is essential for this paper - is still described by Eq. (9). Second, the direct mechanical encounters of grains cannot be treated within the formalism. When such collisions start dominating (at large $T_{d}$ ) the additional dissipation associated with grain deformation, shattering, etc. should be taken into account.

In future, we plan to extend the mechanism for the PAH population of grains, explicitly taking into account the substantial variation of grain charge in the process of individual collision with an electron or ion (remind that in this paper we assumed small charge fluctuations, which allowed us to obtain analytically tractable results). The fact that the efficiency of grain acceleration increases with the decrease of grain size makes the process very efficient. Extrapolating our results to PAH particles we may expect their collisions to be very frequent. We note that the additional motivation for the PAH studies comes from the fact that they are responsible for a component of CMB foreground radiation (Draine \& Lazarian 1998, see also Hoang et al. 2010).

Furthermore, we plan to analyze the impact of the charge-fluctuation-induced acceleration on the dust coagulation processes occurring in the ISM and other astrophysical environments, e.g. protostellar accretion disks. It is already clear that the new mechanism can induce coagulation and shattering which have not been considered in the literature before. Together with the MHD turbulence acceleration, our mechanism testifies that the astrophysical grains achieve velocities much larger than those arising from the Brownian motion.

\section{SUMMARY}

Our major results can be summarized as follows: 1. The change of the grain charge in the process of the grain-grain Coulomb collisions results in second-order Fermi acceleration.

2. The acceleration is most efficient for small, i.e., less than $\sim 10^{-6} \mathrm{~cm}$ grains.

3. The process of acceleration should be accounted for correct description of the evolution of the smallest grains.

AL and TH acknowledge the support of the NSFfunded Center for Magnetic Self-Organizatiaon (CMSO) and the NSF grant AST 0507164.

\section{APPENDIX}

\section{A. COLLISION OPERATOR FOR DUST-GAS INTERACTIONS}

The interaction of individual dust particles with the background neutral gas is described by the Langevin equation (see Van Kampen 1981),

$$
\dot{\mathbf{p}}=-\tau_{d n}^{-1} \mathbf{p}+\mathbf{L}(t)
$$

where $\tau_{d n}^{-1}$ is given by Eq. (11). The random Langevin force has zero average, $\langle\mathbf{L}(t)\rangle=0$, and is properly normalized, $\langle\mathbf{L}(t) \mathbf{L}(t+\tau)\rangle=2 \tau_{d n}^{-1} m_{d} k_{\mathrm{B}} T_{n} \delta(\tau)$, to satisfy the fluctuation-dissipation theorem.

The formalism of the Langevin equation is equivalent to the Fokker-Planck approach which describes evolution of the velocity distribution function (Lifshitz \& Pitaevskii 1981, Van Kampen 1981). The latter approach is solely determined by the first and second Fokker-Plank coefficients, $\mathcal{A}=\langle\delta \mathbf{p}\rangle / \delta t$ and $\mathcal{B}=\left\langle(\delta \mathbf{p})^{2}\right\rangle / 2 \delta t$ (where $\langle\ldots\rangle$ denotes the averaging 
over many collisions occurring over the time period $\delta t$ ). They play the role of the mobility and diffusion coefficients in the velocity space, respectively, and for processes that allow stable equilibrium (e.g., for dust-neutral collisions) they are interrelated. The resulting collision operator for dust-neutral collisions has the following differential form (Lifshitz \& Pitaevskii 1981, Van Kampen 1981),

$$
\mathrm{St}_{d n} f_{d}(\mathbf{p})=\mathcal{B}_{d n} \frac{\partial}{\partial \mathbf{p}}\left(\frac{\mathbf{p}}{m T_{n}} f_{d}+\frac{\partial f_{d}}{\partial \mathbf{p}}\right)
$$

where $\mathcal{B}_{d n}=\tau_{d n}^{-1} m_{d} k_{\mathrm{B}} T_{n}$ is the second Fokker-Plank coefficient for dust-neutral collisions. The integration immediately yields

$$
\int \frac{p^{2}}{m_{d}} \mathrm{St}_{d n} f_{\mathrm{M}} d \mathbf{p}=-6 \tau_{d n}^{-1} k_{\mathrm{B}}\left(T_{d}-T_{n}\right) .
$$

\section{B. COLLISION OPERATOR FOR DUST-DUST INTERACTIONS}

We employ the most general form of the collision operator for binary collisions,

$$
\mathrm{St}_{d} f(\mathbf{p})=\int\left[w\left(\mathbf{p}^{\prime}, \mathbf{p}_{1}^{\prime} ; \mathbf{p}, \mathbf{p}_{1}\right) f\left(\mathbf{p}^{\prime}\right) f\left(\mathbf{p}_{1}^{\prime}\right)-w\left(\mathbf{p}, \mathbf{p}_{1} ; \mathbf{p}^{\prime}, \mathbf{p}_{1}^{\prime}\right) f(\mathbf{p}) f\left(\mathbf{p}_{1}\right)\right] d \mathbf{p}_{1} d \mathbf{p}^{\prime} d \mathbf{p}_{1}^{\prime} .
$$

This form allows for the energy non-conservation in the mutual dust collisions and therefore does not require the unitarity relation to be satisfied [which reduces Eq. (B1) to the canonical Boltzmann form (Lifshitz \& Pitaevskii 1981)]. Here, $w\left(\mathbf{p}, \mathbf{p}_{1} ; \mathbf{p}^{\prime}, \mathbf{p}_{1}^{\prime}\right)$ is a probability function for a pair of colliding particles with momenta $\mathbf{p}$ and $\mathbf{p}_{1}$ to acquire momenta $\mathbf{p}^{\prime}$ and $\mathbf{p}_{1}^{\prime}$, respectively, after the collision. Equation (B1) counts for all possible transitions $\left(\mathbf{p}^{\prime}, \mathbf{p}_{1}^{\prime}\right) \rightarrow\left(\mathbf{p}, \mathbf{p}_{1}\right)$ and then is averaged over $\mathbf{p}_{1}$. Function $w$ can be determined by solving a mechanical problem of the binary scattering with given interaction between the particles.

Assuming the Maxwellian distribution, $f(\mathbf{p})=f_{\mathrm{M}}(\mathbf{p})$, it is convenient to introduce the "binary" distribution function of dust, $F\left(\mathbf{p}_{\mathrm{c}}, \mathbf{p}_{\mathrm{r}}\right) \equiv f_{\mathrm{M}}\left(\mathbf{p}_{1}\right) f_{\mathrm{M}}\left(\mathbf{p}_{2}\right)$. We also define the probability function $W\left(\mathbf{p}_{\mathrm{c}}, \mathbf{p}_{\mathrm{r}} ; \mathbf{q}\right) \equiv w\left(\mathbf{p}, \mathbf{p}_{1} ; \mathbf{p}^{\prime}, \mathbf{p}_{1}^{\prime}\right)$ for the relative momentum exchange $\mathbf{p}_{\mathrm{r}} \rightarrow \mathbf{p}_{\mathrm{r}}+\mathbf{q}$ and after the integration of Eq. (B1) obtain (I05),

$$
\int \frac{p^{2}}{m_{d}} \mathrm{St}_{d d} f_{\mathrm{M}} d \mathbf{p}=\frac{1}{4} \int \frac{p_{\mathrm{r}}^{2}}{m_{d}}\left[W\left(\mathbf{p}_{\mathrm{c}}, \mathbf{p}_{\mathrm{r}}-\mathbf{q} ; \mathbf{q}\right) F\left(\mathbf{p}_{\mathrm{c}}, \mathbf{p}_{\mathrm{r}}-\mathbf{q}\right)-W\left(\mathbf{p}_{\mathrm{c}}, \mathbf{p}_{\mathrm{r}} ; \mathbf{q}\right) F\left(\mathbf{p}_{\mathrm{c}}, \mathbf{p}_{\mathrm{r}}\right)\right] d \mathbf{p}_{\mathrm{c}} d \mathbf{p}_{\mathrm{r}} d \mathbf{q}
$$

In a homogeneous and isotropic medium, the collision probability depends only on the absolute value of the relative momentum, $p_{\mathrm{r}}$, and does not depend on $\mathbf{p}_{\mathrm{c}}$. Thus, $W=W\left(p_{\mathrm{r}} ; \delta q\right)$, where we introduced the absolute value for the inelastic momentum exchange, $\delta q=\left|\mathbf{p}_{\mathrm{r}}+\mathbf{q}\right|-p_{\mathrm{r}}$. For weakly inelastic collisions with $\delta q \ll p_{\mathrm{r}}$ we have

$$
\delta q \approx 2 \frac{m_{d} \delta \varepsilon_{\mathrm{r}}}{p_{\mathrm{r}}} .
$$

Furthermore, the integrand in Eq. (B2) can be expanded into a series over $\delta q$. Retaining the linear and quadratic terms and integrating in parts, we obtain

$$
\int \frac{p^{2}}{m_{d}} \mathrm{St}_{d d} f_{\mathrm{M}} d \mathbf{p} \approx \frac{1}{2 m_{d}} \int\left(p_{\mathrm{r}} \mathcal{A}_{d d}+\mathcal{B}_{d d}\right) f_{\mathrm{M}} d \mathbf{p}_{\mathrm{r}}
$$

where $\mathcal{A}_{d d}\left(p_{\mathrm{r}}\right)=\int \delta q W d \delta q$ and $\mathcal{B}_{d d}\left(p_{\mathrm{r}}\right)=\frac{1}{2} \int(\delta q)^{2} W d \delta q$ are analogous to the Fokker-Planck coefficients for inelastic dust-dust collisions (I05). The coefficients can be expressed in terms of the binary collision cross section $\Sigma$ in the following form:

$$
\begin{aligned}
& \mathcal{A}_{d d}=\int \delta q n_{d} v_{\mathrm{r}} d \Sigma, \\
& \mathcal{B}_{d d}=\frac{1}{2} \int(\delta q)^{2} n_{d} v_{\mathrm{r}} d \Sigma .
\end{aligned}
$$

Using Eqs (6) and (B3) we obtain that $\delta q=O\left(\sigma_{Q}^{2}\right)$. Therefore, $\mathcal{A}_{d d}=O\left(\sigma_{Q}^{2}\right)$ and $\mathcal{B}_{d d}=O\left(\sigma_{Q}^{4}\right)$, i.e., for weakly fluctuating charges the mean energy variation is determined by coefficient $\mathcal{A}_{d d}$ only.

\section{CAlculation of $\mathcal{A}_{D D}$}

In order to calculate the kinetic coefficient $\mathcal{A}_{d d}$, we assume the Coulomb interaction between charged grains (the main contribution to $\mathcal{A}_{d d}$ comes from the interaction at shorter distances, see I05). By substituting $d \varphi_{0} / d r=-Q_{0} / r^{2}$ in Eq. (6) and integrating along the trajectory $r(t)=\sqrt{\rho^{2}+\left(v_{\mathrm{r}} t\right)^{2}}$, we obtain the energy variation $\delta \varepsilon_{\mathrm{r}} \sim\left(\sigma_{Q}^{2} Q_{0}^{2} / \rho^{3} \nu_{\mathrm{ch}} p_{\mathrm{r}}\right)$ (here $\rho$ is the impact parameter of colliding particles, for simplicity we consider the scattering at small angles) and the corresponding momentum variation $\delta q$ [using Eq. (B33)]. Finally, we substitute the result in the first equation (B5), where we use the relation between the scattering angle and the impact parameter in the Coulomb limit, $\chi \sim Q_{0}^{2} / \rho \varepsilon_{\mathrm{r}}$, and employ the Coulomb differential cross section, $d \Sigma \sim\left(Q_{0}^{4} / \varepsilon_{\mathrm{r}}^{2} \chi^{3}\right) d \chi$ (Landau \& Lifshitz 1976). By integrating over 
$0 \leq \chi \leq 1$ we derive,

$$
\mathcal{A}_{d d} \sim \frac{\sigma_{Q}^{2} \omega_{\mathrm{p} d}^{2} p_{\mathrm{r}}}{Q_{0}^{2} \nu_{\mathrm{ch}}}
$$

\section{NUMERICAL ESTIMATE FOR RMS GRAIN VELOCITY}

The ultimate kinetic temperature of grains, $T_{d}^{\infty}$, is given by Eq. (12). It is determined by the plasma Debye length,

$$
\lambda_{\mathrm{D}}=\left(\frac{k_{B} T_{i}}{4 \pi e^{2} n_{i}}\right)^{1 / 2} \approx \frac{63}{n_{i}^{1 / 2}}\left(\frac{T_{i}}{100 \mathrm{~K}}\right)^{1 / 2} \mathrm{~cm},
$$

where $n_{i}$ is in $\mathrm{cm}^{-3}$. Let us assume the MRN size distribution for dust, Eq. (11). Taking into account that the relevant dust density is $n_{d}(a)=\int_{a}^{\infty}\left(d n_{d} / d a\right) d a$, assuming that $n_{n} \sim n_{\mathrm{H}}$, and using Eq. (D1) we get

$$
\frac{T_{d}^{\infty}}{T_{i}} \approx\left(\frac{4 \times 10^{9}}{\alpha n_{i}}\right)\left(\frac{T_{i}}{100 \mathrm{~K}}\right)^{2}\left(\frac{a}{10^{-8} \mathrm{~cm}}\right)^{-3.5},
$$

where the renormalizing factor $\alpha$ for the subsonic and supersonic regimes is given by Eq. (2) and (3), respectively. The corresponding mean squared velocity of grains is

$$
\left(v_{T_{d}}^{\infty}\right)^{2}=\frac{k_{\mathrm{B}} T_{d}^{\infty}}{m_{d}}=T_{d}^{\infty} \times 10^{7}\left(\frac{a}{10^{-8} \mathrm{~cm}}\right)^{-3}(\mathrm{~cm} / \mathrm{s})^{2},
$$

where the dust mass $m_{d}=(4 \pi / 3) \rho a^{3} \approx 10^{-23}\left(a / 10^{-8} \mathrm{~cm}\right)^{3}$ is used. By substituting (D2) into (D3) we finally get

$$
v_{T_{d}}^{\infty} \approx \frac{6 \times 10^{2}}{\left(\alpha n_{i}\right)^{1 / 2}}\left(\frac{T_{i}}{100 \mathrm{~K}}\right)^{3 / 2}\left(\frac{a}{10^{-6} \mathrm{~cm}}\right)^{-3.25} \mathrm{~cm} / \mathrm{s} .
$$

\section{REFERENCES}

Allamandola, L. J., Tielens, A. G. G. M., \& Barker, J. R. 1989, ApJS, 71, 733

de Angelis, U., Ivlev, A. V., Morfill, G. E., \& Tsytovich, V. N. 2005, Phys. Plasmas, 12, 052301

Cho, J., \& Lazarian, A. 2003, MNRAS, 345, 325

Cho, J., \& Lazarian, A. 2002, Physical Review Letters, 88, 245001

Draine, B. T. 2009, EAS Publications Series, 35, 245

Draine, B. T., \& Lee, H. M. 1984, ApJ, 285, 89

Draine, B. T., \& Li, A. 2007, ApJ, 657, 810

Draine, B. T. 1985, Protostars and Planets II, 621

Draine, B. T. 2003, ARA\&A, 41, 241

Draine, B. T., \& Sutin, B. 1987, ApJ, 320, 803

Draine, B. T., \& Salpeter, E. E. 1979, ApJ, 231, 438

Fortov, V. E., Ivlev, A. V., Khrapak, S. A., Khrapak, A. G., \& Morfill, G. E. 2005, Phys. Rep., 421, 1

Fraisse, A. A., et al. 2009, American Institute of Physics Conference Series, 1141, 265

Goree, J. 1994, Plasma Sources Sci. Technol., 3, 400

Hoang, T., Draine, B. T., \& Lazarian, A. 2010, ApJ, 715, 1462

Ivlev, A. V., Zhdanov, S. K., Klumov, B. A., Tsytovich, V. N., de Angelis, U., Morfill, G. E. 2004, Phys. Rev. E, 70, 066401

Ivlev, A. V., Zhdanov, S. K., Klumov, B. A., \& Morfill, G. E. 2005, Phys. Plasmas 12, 092104

Khrapak, S. A., Nefedov, A. P., Petrov, O. F., \& Vaulina, O. S. 1999, Phys. Rev. E 59, 6017

Landau, L. D., \& Lifshitz, E. M. Mechanics (Pergamon, Oxford, 1976)
Lazarian, A., \& Finkbeiner, D. 2003, New Astronomy Review, 47, 1107

Lazarian, A., \& Yan, H. 2002, ApJ, 566, L105

Leger, A., \& Puget, J. L. 1984, A\&A, 137, L5

Lifshitz, E. M., \& Pitaevskii, L. P. Physical Kinetics (Pergamon, Oxford, 1981)

Mathis, J. S., Rumpl, W., \& Nordsieck, K. H. 1977, ApJ, 217, 425

Matsoukas, T., \& Russell, M. 1997, Phys. Rev. E, 55, 991

Purcell, E. M. 1969, Physica, 41, 100

Tielens, A. G. G. M. 2005, The Physics and Chemistry of the

Interstellar Medium, by A. G. G. M. Tielens, pp. . ISBN 0521826349. Cambridge, UK

Tsytovich, V. N. Phys. Usp. 1997, 40, 53

Tsytovich, V. N., Morfill, G. E., Vladimirov, S. V., \& Thomas, H. M. Elementary physics of complex plasmas (Springer, Berlin, 2008)

Spitzer, L. Physical Processes in the interstellar medium (New

York, 1978)

van Kampen, N. G. Stochastic Processes in Physics and Chemistry (Elsevier, Amsterdam, 1981)

Yan, H., Lazarian, A., \& Draine, B. T. 2004, ApJ, 616, 895

Yan, H., \& Lazarian, A. 2003, ApJ, 592, L33

Weingartner, J. C., \& Draine, B. T. 2001, ApJ, 563, 842

D. C. B. Whittet, Dust in the Galactic Environment (IOP Publ., Bristol, 2003). 